\title{
Two-dimensional hydrogen-like atom in a uniform magnetic field. Large-order perturbation theory
}

\author{
Francisco M. Fernández * \\ INIFTA, División Química Teórica \\ Blvd. 113 S/N, Sucursal 4, Casilla de Correo 16, 1900 La Plata, Argentina
}

\begin{abstract}
The well known hypervirial perturbation method (HPM) based on hypervirial relations and the Hellmann-Feynman theorem is suitable for the calculation of perturbation corrections of large order for the twodimensional hydrogen-like atom in a uniform magnetic field. We show analytical results in terms of the quantum numbers and large order corrections for particular states. This approach appears to be simpler and more efficient than the recently proposed one based on Green functions.
\end{abstract}

\section{Introduction}

The two-dimensional hydrogen-like atom in a uniform magnetic field perpendicular to the atomic plane has received considerable attention as a model for excitons in thin materials such as nano-scale multilayer semiconductor systems. The Schrödinger equation is separable in polar coordinates and has been solved approximately in many different ways [1]13. Here we are interested in the application of perturbation theory that provides approximate solutions in terms of power series of the field strength that are suitable in the weak-field limit [3, 8, 10,13.

*E-mail: fernande@quimica.unlp.edu.ar 
Several authors have obtained perturbation corrections of low order [3, 8, 10,11,13 but the most extended calculation was due to Szmytkowski [13] who solved the perturbation equations of first and second order by means of Green functions. His analytical results, which are valid for all quantum numbers, suggest that some of the earlier results [10 12] may be wrong. The Greenfunction method is a powerful approach that requires considerable ingenuity and mastery of the technique; however, it appears to be unsuitable for the calculation of perturbation corrections of larger order because it soon becomes rather intractable.

There are other strategies for the calculation of the perturbation corrections for simple quantum-mechanical models that are more efficient [14 and the purpose of this paper is to discuss one of them. Taking into account the interest in the two-dimensional hydrogen-like atom in a magnetic field and the discrepancy between earlier perturbation calculations, present analysis appears to be fully justified.

In section 2 we outline the model and derive a dimensionless eigenvalue equation for the radial part of the Schrödinger equation that is suitable for the application of perturbation theory. In section 3 we briefly develop the chosen perturbation technique and apply it to the model mentioned above. Finally, in section 4 we summarize the main results of the paper and draw conclusions.

\section{Model}

The model consists of a two-dimensional hydrogen-like atom in a uniform magnetic field perpendicular to the atomic plane. It may also represent the effectivemass equation for an electron-hole pair in the presence of a uniform magnetic field 1, 2]. For concreteness, in what follows we resort to the notation of Szmytkowski [13] in order to facilitate comparison of the results. In the case of a symmetric gauge the Schrödinger equation is separable in polar coordinates 
$(0 \leq r<\infty, 0 \leq \phi<2 \pi)$. If we write the eigenfunctions as

$$
\psi(r, \phi)=\frac{1}{\sqrt{r}} P(r) \frac{1}{\sqrt{2 \pi}} e^{i m_{l} \phi}, m_{l}=0, \pm 1, \pm 2, \ldots,
$$

the radial part of the Schrödinger equation becomes [13]

$$
\left[-\frac{\hbar^{2}}{2 m} \frac{d^{2}}{d r^{2}}+\frac{\hbar^{2}\left(l^{2}-1 / 4\right)}{2 m r^{2}}+\frac{e \hbar B}{2 m} m_{l}-\frac{Z e^{2}}{4 \pi \epsilon_{0} r}+\frac{e^{2} B^{2} r^{2}}{8 m}\right] P(r)=E P(r)
$$

where $m$ and $-e$ are the electron mass and charge, respectively, $Z e$ is the charge of the spinless nucleus (clamped at the coordinate origin), $l=\left|m_{l}\right|$ and $B$ is the field intensity. The bound-state solutions satisfy

$$
\lim _{r \rightarrow 0} P(r)=0, \lim _{r \rightarrow \infty} P(r)=0 .
$$

Straightforward application of perturbation theory to equation (2) is cumbersome because one has to carry all the physical constants and model parameters through the whole algebraic process [13]. For this reason it is preferable to convert equation (2) into a dimensionless eigenvalue equation. To this end we define a dimensionless coordinate $q=r / L$, where

$$
L=\frac{a_{0}}{Z}, a_{0}=\frac{4 \pi \epsilon_{0} \hbar^{2}}{m e^{2}}
$$

and equation (2) becomes

$$
\begin{aligned}
H P(r) & =\epsilon P(r), \\
H & =\left[-\frac{1}{2} \frac{d^{2}}{d q^{2}}+\frac{\xi}{2 q^{2}}-\frac{1}{q}+\lambda q^{2}\right],
\end{aligned}
$$

where

$$
\begin{aligned}
\epsilon & =\frac{m L^{2}}{\hbar^{2}}\left(E-\frac{e \hbar B}{2 m} m_{l}\right), \\
\xi & =l^{2}-\frac{1}{4}, \lambda=\frac{e^{2} B^{2} L^{2}}{8 \hbar^{2}} .
\end{aligned}
$$

The unit of energy is

$$
\frac{\hbar^{2}}{2 m L^{2}}=\frac{Z e^{2}}{4 \pi \epsilon_{0} L}=\frac{Z^{2} e^{2}}{4 \pi \epsilon_{0} a_{0}}
$$

and

$$
\frac{e \hbar B}{2 m} \frac{m L^{2}}{\hbar^{2}}=\sqrt{2 \lambda}
$$


If we define

$$
B_{0}=\frac{\hbar}{e a_{0}}
$$

then

$$
\lambda=\frac{1}{8 Z^{4}}\left(\frac{B}{B_{0}}\right)^{2},
$$

enables us to compare present results with those of Szmytkowski [13]. By means of perturbation theory we easily obtain the coefficients of the perturbation series

$$
\epsilon(\lambda)=\sum_{p=0}^{\infty} \epsilon_{p} \lambda^{p}
$$

When $B=0(\lambda=0)$ the problem is exactly solvable and the allowed energies are given by 13

$$
\epsilon_{0}=-\frac{1}{2\left(n_{r}+l+1 / 2\right)^{2}}
$$

where $n_{r}=0,1, \ldots$ is the radial quantum number (number of zeroes of $P(r)$ ). Following previous papers we also define $n=n_{r}+l+1$ that resembles the principal quantum number of the three-dimensional hydrogen atom.

The weak-field series (111) is divergent. If we define the new variable $u=$ $\lambda^{1 / 4} q$ then the dimensionless operator $H$ becomes

$$
H=\sqrt{\lambda}\left(-\frac{1}{2} \frac{d^{2}}{d u^{2}}+\frac{\xi}{2 u^{2}}-\frac{\lambda^{-1 / 4}}{u}+u^{2}\right)
$$

that clearly shows that we can also expand the eigenvalues in the strong-field series

$$
\epsilon=\sqrt{\lambda} \sum_{j=0}^{\infty} e_{j} \lambda^{-j / 4}
$$

which has a finite radius of convergence.

\section{$3 \quad$ Hypervirial perturbation method}

One of the most efficient implementations of perturbation theory for separable problems is the hypervirial perturbation method (HPM) that combines the hypervirial relations and the Hellmann-Feynman theorem. If $O$ is a linear operator then

$$
\langle P|[H, O]| P\rangle=0,
$$


is called a hypervirial relation, where $[H, O]=H O-O H$ is the commutator between such pair of operators [14]. This equation holds if $O$ is chosen so that $\langle P \mid H O P\rangle=\langle H P \mid O P\rangle$. Another important ingredient of the method is the Hellmann-Feynman theorem that states that [14]

$$
\frac{\partial \epsilon}{\partial \lambda}=\left\langle\frac{\partial H}{\partial \lambda}\right\rangle \text {. }
$$

If

$$
H-\frac{1}{2} \frac{d^{2}}{d q^{2}}+\frac{\xi}{2 q^{2}}-\frac{1}{q}+\lambda q^{K}, K=1,2, \ldots,
$$

and $O=\frac{j+1}{2} q^{j}-q^{j+1} \frac{d}{d q}$ [14 then equation (15) becomes

$$
\begin{aligned}
& 2 j \epsilon Q_{j-1}+(j-1)\left[\frac{j(j-2)}{4}-\xi\right] Q_{j-3}+(2 j-1) Q_{j-2}-(2 j+K) \lambda Q_{j+K-1}=0 \\
& Q_{j}=\left\langle q^{j}\right\rangle, j=1,2, \ldots
\end{aligned}
$$

On expanding the expectation values in a Taylor series

$$
Q_{j}=\sum_{p=0}^{\infty} Q_{j, p} \lambda^{p}
$$

we obtain

$$
\begin{aligned}
Q_{j, i}= & \frac{1}{2(j+1) \epsilon_{0}}\left\{j\left[\xi-\frac{j^{2}-1}{4}\right] Q_{j-2, i}-(2 j+1) Q_{j-1, i}\right. \\
& \left.-2(j+1) \sum_{m=1}^{i} \epsilon_{m} Q_{j, i-m}+(2 j+K+2) Q_{j+K, i-1}\right\}, \\
j= & 1,2, \ldots
\end{aligned}
$$

In addition to this equation we also have

$$
Q_{-1, i}=-2 \epsilon_{i}+(K+2) Q_{K, i-1},
$$

that comes from (18) with $j=1$. It is assumed that $Q_{0}=1$, so that $Q_{0, i}=\delta_{i 0}$ is a starting point for the recurrences.

In order to obtain the perturbation coefficients $\epsilon_{i}$ and $Q_{j, i}$ we need an additional equation provided by the Hellmann-Feynman theorem (16)

$$
\epsilon_{i}=\frac{1}{i} Q_{K, i-1}, i=1,2, \ldots
$$


The calculation is straightforward and for $K=2$ (present case) we obtain

$$
\begin{gathered}
\epsilon_{1}=\frac{(2 n-1)^{2}}{8}\left(-3 l^{2}+3+5 n^{2}-5 n\right), \\
\epsilon_{2}=-\frac{(2 n-1)^{6}}{1024}\left(-21 l^{4}-138 l^{2}+159-90 l^{2} n^{2}+90 l^{2} n+582 n^{2}-439 n+143 n^{4}-286 n^{3}\right) \\
\epsilon_{3}=\frac{(2 n-1)^{10}}{65536}\left(17967-65495 n+68835 n^{4}-107070 n^{3}+115970 n^{2}-18066 l^{2}-35130 l^{2} n^{2}\right. \\
\left.\quad+29910 l^{2} n+231 l^{4}-132 l^{6}-18360 n^{5}+6120 n^{6}+10440 l^{2} n^{3}-5220 l^{2} n^{4}\right) \\
\epsilon_{4}=\quad-\frac{(2 n-1)^{14}}{16777216}\left(15522195-67825511 n-4005 l^{8}+153888490 n^{4}-180523168 n^{3}+145662172 n^{2}\right. \\
\quad-17506020 l^{2}-64292340 l^{2} n^{2}+43194060 l^{2} n+1991850 l^{4}-4020 l^{6}-80558702 n^{5}+33863592 n^{6} \\
+43836660 l^{2} n^{3}-26018580 l^{2} n^{4}-1640100 l^{2} n^{6}+4920300 l^{2} n^{5}-502740 l^{4} n^{3}+2563680 l^{4} n^{2} \\
\end{gathered}
$$

The first three perturbation corrections agree with those of Szmytkowski [13] to which we added $\epsilon_{4}$ that was not reported before as far as we know. We can obtain as many perturbation corrections as desired by means of the recurrence relations (201), (21) and (22) and available computer algebra software. However, the perturbation corrections in terms of the quantum numbers may probably be too long for any practical use. If one is interested in weak-field expansions of large order it appears to be preferable to obtain them for particular values of $n$ and $l$ be means of those recurrence relations. For example, when $n=1$ and $l=0$ we have

$$
\begin{aligned}
\epsilon_{1} & =3 / 8, \epsilon_{2}=-\frac{159}{1024}, \epsilon_{3}=\frac{17967}{65536}, \epsilon_{4}=-\frac{15522195}{16777216}, \epsilon_{5}=\frac{5189052801}{1073741824} \\
\epsilon_{6} & =-\frac{4896676641339}{137438953472}, \epsilon_{7}=\frac{3094900497137871}{8796093022208}, \epsilon_{8}=-\frac{20233178231139761499}{4503599627370496}, \\
\epsilon_{9} & =\frac{20808558827825859998445}{288230376151711744}, \epsilon_{10}=-\frac{52693485465369543566065089}{36893488147419103232}, \\
\epsilon_{11} & =\frac{80639435078901048406195920633}{2361183241434822606848}, \epsilon_{12}=-\frac{587353055515797037508553136130823}{604462909807314587353088}, \\
\epsilon_{13} & =\frac{1255613239147236284205667622925365349}{38685626227668133590597632}
\end{aligned}
$$




$$
\begin{aligned}
\epsilon_{14} & =-\frac{6229668057619980010555555519950165544755}{4951760157141521099596496896} \\
\epsilon_{15} & =\frac{17753264589549239693872523415436400485638255}{316912650057057350374175801344} \\
\epsilon_{16} & =-\frac{921721759137179716887942948086717222595277533675}{324518553658426726783156020576256} \\
\epsilon_{17} & =\frac{3379056665253674076167201632469154672196055608756005}{20769187434139310514121985316880384} \\
\epsilon_{18} & =-\frac{27797116247667972439940810526714208588100705850127986405}{2658455991569831745807614120560689152} \\
\epsilon_{19} & =\frac{127484555261829518463134910686385252583016203699835125715445}{170141183460469231731687303715884105728} \\
\epsilon_{20} & =-\frac{2593203450314371618931792865686398116783507010792581025252777725}{43556142965880123323311949751266331066368}
\end{aligned}
$$

It is worth noting that the HPM cannot be applied to the operator (13) for the calculation of the strong-field series. The reason is that the unperturbed operator $H_{0}=\lim _{\lambda \rightarrow \infty} \lambda^{-1 / 2} H$ is an even function of the coordinate $u$ and therefore the recurrence relations do not yield the expectation values $U_{j}=\left\langle u^{j}\right\rangle$ for odd and even $j$ necessary for the application of the technique and the calculation of $U_{-1}$.

\section{Conclusions}

The calculation of perturbation corrections of large order by means of Greens functions does not appear to be practicable. On the other hand, the HPM yields simple straightforward recurrence relations for the systematic calculation of the perturbation corrections to the energy and expectation values of powers of the coordinate. These recurrence relations are suitable for programming in available computer algebra systems which greatly facilitates the systematic calculation of those perturbation corrections to any desired order.

Present results agree with those of Szmytkowski [13] and not with those derived by other authors by means of the Levi-Civita transformation of the coordinates and the expression of the resulting two-dimensional Hamiltonian operator in terms of suitable creation-annihilation operators [10 12. We have no doubt that their weak-field expansions do not agree with the actual ones. For 
example, the weak-field expansions derived by Hoang et al [12] are not correct because they come from approximate variational expressions for the energies. However, it is worth mentioning that for sufficiently large field intensities they fit the actual eigenvalues more accurately than the exact weak-field expansions because the latter are divergent.

\section{References}

[1] O. Akimoto and H. Hasegawa, Interband optical transitions in extremely anysotropic semiconductors. II Coexistence of excitons and the Landau levels, J. Phys. Soc. Jpn 22 (1967) 181-191.

[2] M. Shinada and K. Tanaka, Interband optical transitions in extremely anisotropic semiconductors. III. Numerical studies of magneto-optical absorption, J. Phys. Soc. Jpn 29 (1970) 1258-1268.

[3] A. H. MacDonald and Ritchie. D. S., Hydrogenic energy levels in two dimensions at arbitrary magnetic fields, Phys. Rev. B 33 (1986) 8336-8344.

[4] W. Edelstein, H. N. Spector, and R. Marasas, Two-dimensional excitons in magnetic fields, Phys. Rev. B 39 (1989) 7697-7703.

[5] J.-L. Zhu, Y. Cheng, and J.-J. Xiong, Quantum levels and Zeeman splitting for two-dimensional hydrogenic donor states in a magnetic field, Phys. Rev. B 41 (1990) 10792-10798.

[6] P. Martín, J. J. Rodriguez-Nuñez, and J. L. Marquez, Two-dimensional hydrogenlike atoms in the presence of a magnetic field: Quasifractional approximations, Phys. Rev. B 45 (1992) 8359-8362.

[7] L. V. Hoang and N. T. Giang, The algebraic method for two-dimensional quantum atomic systems, J. Phys. A 26 (1993) 1409-1418.

[8] M. Robnik and V. G. Romanovski, Two-dimensional hydrogen atom in a strong magnetic field, J. Phys. A 36 (2003) 7923-7951. 
[9] N.-T. Hoang-Do, D.-L. Pham, and V.-H. Le, Exact numerical solutions of the Schrödinger equation for a two-dimensional exciton in a constant magnetic field of arbitrary strength, Physica B 423 (2013) 31-37.

[10] N.-T. Hoang-Do, V.-H. Hoang, and V.-H. Le, Analytical solutions of the Schrödinger equation for a two-dimensional exciton in magnetic field of arbitrary strength, J. Math. Phys. 54 (2013) 052105.

[11] I. Feranchuk, A. Ivanov, V.-H. Le, and A. Ulyanenkov, Non-perturbative Description of Quantum Systems, Springer, Cham, (2015).

[12] N.-T. D. Hoang, D.-A. P. Nguyen, V.-H. Hoang, and V.-H. Le, Highly accurate analytical energy of a two-dimensional exciton in a constant magnetic field, Physica B 495 (2016) 16-20.

[13] R. Szmytkowski, Two-dimensional hydrogen-like atom in a weak magnetic field, 2018. arXiv:1806.03477 [quant-ph].

[14] F. M. Fernández, Introduction to Perturbation Theory in Quantum Mechanics, CRC Press, Boca Raton, (2000). 